\title{
A utilização de modelos de simulação para treinamento cirúrgico - revisão integrativa
}

\author{
The use of simulation models for surgical training - integrative review
}

El uso de modelos de simulación para el entrenamiento quirúrgico - revisión integradora

Nathalia Elen Barbosa dos Santos ORCID: https://orcid.org/0000-0001-7270-2165 Faculdade Paulo Picanço, Brasil E-mail: nathaliaebsantos@gmail.com

Eduardo Brito de Lima

ORCID: https://orcid.org/0000-0002-7533-9098

Faculdade Paulo Picanço, Brasil

E-mail: edubriito15@gmail.com

Marina Araújo Sales

ORCID: https://orcid.org/0000-0002-7348-5913

Faculdade Paulo Picanço, Brasil

E-mail:marinasalesaraujo18@gmail.com

Andrê Parente de Sá Barreto Vieira

ORCID: https://orcid.org/0000-0001-5523-7599

Faculdade Paulo Picanço, Brasil

E-mail:andre.barreto@facpp.edu.br

Radamés Bezerra Melo

ORCID: https://orcid.org/0000-0001-5466-5698

Faculdade Paulo Picanço, Brasil

E-mail: radamesbmelo@ hotmail.com

Carlos Diego Lopes Sá

ORCID: https://orcid.org/0000-0002-7501-4170

Faculdade Paulo Picanço, Brasil

E-mail: carlos.diego@facpp.edu.br

\begin{abstract}
Resumo
Objetivo: O objetivo desse estudo é realizar uma revisão integrativa sobre a utilização dos modelos disponíveis no mercado, abordando questões com estudos já existentes, mostrando os diversos modelos disponíveis, pensando também sua na eficiência e custo benefício. Metodologia: Realizou-se uma busca de artigos nas bases de dados PubMed e Scielo, usando os descritores: simulation-based surgical training, maxillofacial e surgery, sem restringir o idioma. Foram excluídos os artigos que não apresentaram clareza na metodologia e que não se adequavam à temática pesquisada. Resultados: Após a leitura dos resumos e aplicação dos critérios de inclusão e exclusão resultou na seleção de sete artigos para revisão. Os estudos comprovam a eficiência satisfatória dos modelos descritos. Considerações finais: Os modelos facilitam o treinamento nas técnicas cirúrgicas, o que posteriormente pode reduzir o tempo de operação e levar a uma melhor execução das habilidades na realidade.
\end{abstract}

Palavras-chave: Treinamento cirúrgico baseado em simulação; Cirurgia maxilofacial; Cirurgia; Ensino.

\begin{abstract}
Objective: The objective of this study is to carry out a integrative review on the use of models available on the market, addressing issues with existing studies, showing the various models available, also thinking about their efficiency and cost benefit. Methodology: A search of articles in the PubMed and Scielo databases was performed using the descriptors: simulation-based surgical training, maxillofacial and surgery, without restricting the language. Articles that did not present clarity in the methodology and that were not appropriate to the subject researched were excluded. Results: After reading the abstracts and applying the inclusion and exclusion criteria, seven articles were selected for review. The studies proved the satisfactory efficiency of the described models. Final considerations: The models facilitate training in surgical techniques, which can later reduce the operation time and lead to a better execution of skills in reality.
\end{abstract}

Keywords: Simulation-based surgical training; Maxillofacial surgery; Teaching.

\section{Resumen}

Objetivo: El objetivo de este estudio es realizar una revisión integradora sobre el uso de los modelos disponibles en el mercado, abordando los problemas con los estudios existentes, mostrando los diversos modelos disponibles, pensando 
también en su eficiencia y costo beneficio. Metodología: Se realizó una búsqueda de artículos en las bases de datos PubMed y Scielo utilizando los descriptores: simulation-based surgical training, maxillofacial and surgery, sin restringir el idioma. Se excluyeron los artículos que no presentaban claridad en la metodología y no eran apropiados para el tema. Resultados: Tras leer los resúmenes y aplicar los criterios de inclusión y exclusión, se seleccionaron siete artículos para su revisión. Los estudios demostraron la eficacia satisfactoria de los modelos descritos. Observaciones finales Los modelos facilitan el entrenamiento en técnicas quirúrgicas, lo que posteriormente puede reducir el tiempo de la operación y conducir a una mejor ejecución de las habilidades en la realidad.

Palabras clave: Formación quirúrgica basada en la simulación; Cirugía maxilofacial; Cirugía; Ensenãnza.

\section{Introdução}

Os procedimentos cirúrgicos exigem dos médicos e cirurgiões-dentistas várias habilidades, dentre elas a precisão, para o momento da intervenção nos pacientes. Para que não haja nenhuma complicação durante os procedimentos a serem executados, é necessário que os profissionais possuam um conhecimento exato sobre cada intervenção, dessa maneira os riscos, através de complicações que possam surgir, diminuem consideravelmente. (Carvalho et al., 2019; Ahmed et al., 2018; Kumaresan et al., 2013).

Um dos exemplos relacionados à necessidade do conhecimento e autoconfiança dos profissionais no momento dos procedimentos é a cirurgia oral e maxilo-facial, que engloba uma variedade de procedimentos cirúrgicos envolvendo a maioria dos tecidos duros e moles da região craniofacial; exigindo assim, habilidades cirúrgicas, vindas da junção entre teoria e prática. (Carvalho et al., 2018; Werz et al., 2018; Lambrecht et al., 2010).

Nos últimos anos, a simulação cirúrgica tem ganhando um importante espaço na educação de profissionais ligados a saúde. A prática vem sendo utilizada no ambiente acadêmico para planejar e simular uma cirurgia, fazendo com que os estudantes possam realizar um trabalho repetitivo, melhorando sua capacidade analítica-situacional e diminuindo o tempo dos procedimentos sem comprometer o paciente. Tais habilidades acabam preparando o profissional para diferentes situações clínicas encontradas no cotidiano. (Carvalho et al., 2018; Werz et al., 2018; O'callaghan et al., 2017).

Algumas técnicas mesmo sendo utilizado há décadas, como a que utiliza resinas acrílicas endurecidas por laser, ou mesmo modelos 3D de filamentos com borracha de silicone, simulando tecido mole; são alternativas bastante viáveis para o aprendizado. Porém, o custo financeiro acaba sendo uma dificuldade para a sua utilização no ambiente universitário. (Ahmed et al., 2018; Lambrecht et al., 2010; Mccaslin et al., 2011).

Deste modo, o objetivo desse estudo é realizar uma Revisão de Literatura sobre a utilização dos modelos de simulação cirúrgica disponíveis na literatura científica, mostrando os diversos modelos disponíveis, pensando também sua na eficiência e custo benefício.

\section{Metodologia}

O presente estudo trata-se de um estudo qualitativo, descritivo, do tipo revisão (Pereira et al., 2018). A pesquisa foi realizada através de bases de dados fornecidas pelos sites de pesquisas PubMed e SciELO, buscando estudos publicados sem restrições de ano e idioma, com os descritores: "simulation", "Maxilofacial", "Surgery". Foram excluídos os artigos que não se adequavam a temática pesquisada. Vale ressaltar que também foi incluída nesse estudo uma pesquisa manual da lista de referência de artigos selecionados. A busca dos artigos está descrita no fluxograma. 
Figura 1: Fluxograma descreve os critérios de inclusão e exclusão para a busca de artigos adotado nesse estudo.

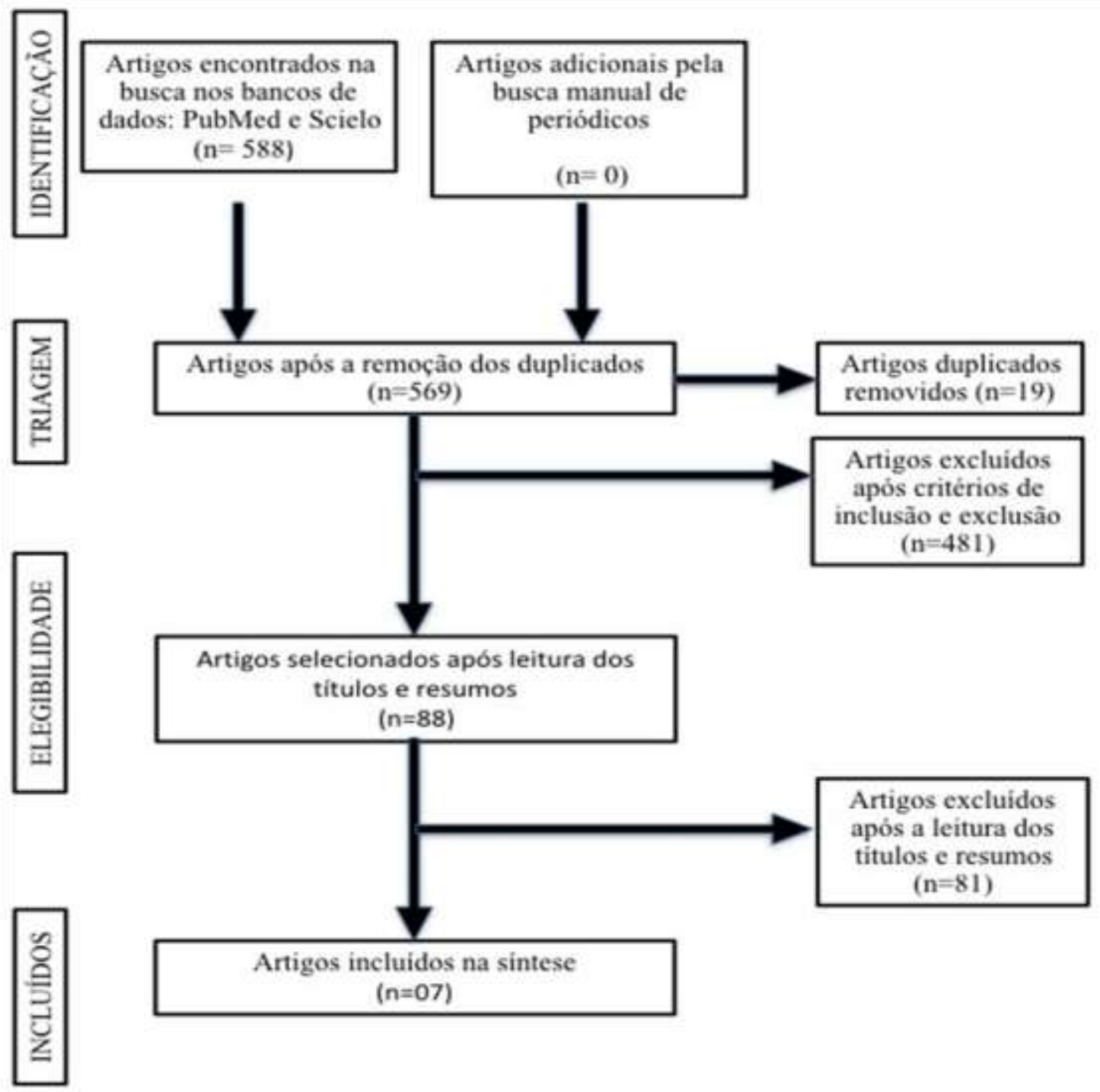

Fonte: Autores.

Inicialmente os artigos foram selecionados por título e resumo de acordo com a estratégia de pesquisa descrita. Artigos encontrados em mais de uma base de dados foram desclassificados. Os resultados que mais condiziam com objetivos dessa pesquisa tiveram os resumos lidos, caso os dados obtidos através do resumo fossem insuficientes, optou-se por ler o artigo completo.

\section{Resultados}

Os estudos comprovam que os usos de modelos de ensino permitem que os acadêmicos aprendam habilidades cirúrgicas sem comprometer o paciente, possibilitando os alunos a lidar com os instrumentais cirúrgicos em um campo limitado sob uma circunstância semi realista e também ajudando no desenvolvimento de habilidades psicomotoras em uma situação não estressante. (Ahmed et al., 2018; Kumaresan et al., 2013). 
Tabela 1: Os principais achados dos artigos selecionados.

\begin{tabular}{|c|c|c|}
\hline ESTUDO & OBJETIVO & PRINCIPAIS ACHADOS \\
\hline Lambrecht et al., 2010 & $\begin{array}{l}\text { Fabricação de modelos tridimensionais } \\
\text { (3D) hápticas utilizando materiais } \\
\text { límpidos e opacos. }\end{array}$ & $\begin{array}{l}\text { A geração de modelos } 3 \mathrm{D} \text { baseados } \\
\text { em conjuntos de dados tomográficos } \\
\text { podem se tornar uma técnica útil para } \\
\text { demonstrar, planejar e auxiliar no } \\
\text { ensino. O objetivo é usar esses } \\
\text { modelos rotineiramente como método } \\
\text { alternativo na educação básica e } \\
\text { avançada, já que a produção em } \\
\text { massa destes modelos é possível. }\end{array}$ \\
\hline Uppal et al., 2012 & $\begin{array}{l}\text { Descrever a utilização de modelo de } \\
\text { sutura de baixo custo confeccionado com } \\
\text { esponja de espuma sintética. }\end{array}$ & $\begin{array}{l}\text { A utilização do modelo melhoraria a } \\
\text { destreza do treinamento cirúrgico, } \\
\text { assim minimizando a incidência de } \\
\text { acidentes com agulhas e obtendo } \\
\text { melhores resultados. A } \\
\text { disponibilidade fácil de espuma e } \\
\text { material do elastômero de impressão, } \\
\text { em conjunto com a preparação rápida, } \\
\text { tornam este modelo de sutura } \\
\text { adequado quando uma solução de } \\
\text { baixo custo é desejada. }\end{array}$ \\
\hline
\end{tabular}

Kolozsvari et al., 2011

Kumaresan et al., 2013

Kumaresan et al., 2013

Descrever a construção de um modelo de sutura simples e de baixo custo para o treinamento pré-clínico.

Analisar a aplicação do desempenho do modelo especializado em cirurgia e o papel da simulação na aquisição de habilidades cirúrgicas.

\begin{tabular}{l|l} 
& \multicolumn{1}{|c}{ treinamento pré-clínico. } \\
\hline
\end{tabular}

Carvalho et al., 2018

Apresentar uma alternativa, simples e de baixo custo como método educativo de formação mucoperioteal e suturas em cirurgia oral e maxilo-facial.

Werz et al., 2018

\begin{tabular}{c}
$\begin{array}{c}\text { habilidades cirúrgicas para estudantes de } \\
\text { odontologia ou residentes de cirurgia oral } \\
\text { e maxilo-facial. }\end{array}$ \\
\hline
\end{tabular}

Avaliar se o modelo 3D de baixo custo pode ser adequado para treinar habilidades cirúrgicas para estudantes de e maxilo-facial.

Ahmed et al., 2018
Rever o atual uso de simulação no treinamento em cirurgia maxilo-facial.
A simulação está rapidamente se tornando um dos pilares da formação de competências cirúrgicas, motivado pelo rápido desenvolvimento de novas tecnologias, incentivando a repetição orientada seguida de feedback, permitindo assim a aprendizagem de tarefas complexas.

A deficiência do modelo descrito é compensada pela produção simples, de baixo custo e a possibilidade de produção em massa, oferecendo procedimentos cirúrgicos idênticos aos estudantes.

O presente método tem uma metodologia simples e de baixo custo, rápido para executar, fácil reprodutibilidade, e reutilizável, uma vez que é possível reutilizar a pedra lançada por escolha de outro material de impressão elástica como um propósito educacional para repetição.

O estudo demonstrou que a impressão em 3D com filamento de impressão de baixo custo é o método promissor para a formação de residentes de cirurgia oral e maxilo-facial e estudantes em procedimentos cirúrgicos.

Esse acesso aos métodos deve melhorar o treinamento cirúrgico para os estagiários de cirurgia, pois são modelos de suporte e beneficiam o treinamento na simulação cirúrgica, então precisamos usar e melhorar os recursos de treinamento atuais.

Fonte: Autores. 


\section{Discussão}

Recentes pesquisas referentes aos modelos de simulação utilizados nos treinamentos cirúrgicos, realizados em estudantes, revelam que o uso de métodos práticos melhora os resultados finais dos procedimentos que devem ser executados pelos acadêmicos. A aplicação de modelos, como nos protótipos descritos nos estudos de Lambrecht et al. 2010 e Werz et al. 2018, obtiveram relevância no meio acadêmico corroborando a tese de que a prática de tais métodos no ambiente de estudo auxilia na redução do tempo da curva de aprendizado técnico. (Wanibuchi et al., 2016; Ilburn et al., 2012).

Tais conclusões encontram base a partir de pesquisas feitas ainda na década de 1980, onde os profissionais habilitados para a realização dos procedimentos cirúrgicos deram início a utilização técnicas em protótipos conhecidos como "phantom cabeças", uma espécie de cabeças humanas feitas de diversos materiais, que ao remeter a um paciente tornavam a experiência ainda mais próxima ao real. (Ahmed et al., 2018; Lambrecht et al., 2010; Lambrecht et al., 1990)

Outros resultados da utilização de métodos prévios antes dos procedimentos cirúrgicos, ainda nos anos de 1980, foi o uso dos primeiros modelos de fabricação tecnológica baseado a laser, utilizados para planejar e simular cirurgia maxilocraniofacial em três dimensões diferentes. (Ahmed et al., 2018; Lambrecht et al., 2010; Lambrecht et al., 1990).

Essa formação, que se revela histórica, baseada em simuladores permite que os alunos possam aprimorar suas habilidades cirúrgicas sem comprometer o paciente. Dessa maneira, qualquer atividade que tenha como objetivo imitar um sistema ou ambiente, com a finalidade de avaliar, informar e modificar habilidades e comportamentos; vem tornando-se cada vez mais comum no ambiente de estudos. (Ahmed et al., 2018; Kumaresan et al., 2013; Ilburn et al., 2012).

Dessa maneira, os resultados encontrados nas pesquisas analisadas sobre os benefícios dos treinamentos através de simulações são, em sua maioria, positivos. Porém, mesmo com os dados favoráveis, os pesquisadores frisam que a adoção deste método é apenas um complemento e não um substituto para o treinamento cirúrgico clínico. (Lambrecht et al., 1990; Uppal et al., 2012; Stefanidis et al., 2010; Ploch et al., 2016).

Levando em consideração fatores econômicos, os modelos de negócios sugerem que financeiramente existe uma vantagem para os hospitais e centros acadêmicos em investir no treinamento de simulação. As técnicas reduzem o tempo de execução e espera e além de diminuir os recursos necessários para treinar os cirurgiões, aumentando a eficiência e a produtividade. A partir destes resultados, a probabilidade de complicações, principalmente no início da curva de aprendizado, diminui consideravelmente. (Mccaslin et al., 2011; Stefanidis et al., 2010; Cohen et al., 2010).

Um dos exemplos de treinamento por simuladores que comprovadamente beneficiam os estudantes, levando em consideração a curva de aprendizado; disponíveis no mercado que podemos utilizar nesta pesquisa é a técnica de sutura. Porém, é comum que materiais e procedimentos ligados a esta prática sejam de alto custo financeiro, o que muitas vezes se torna um empecilho na hora de se obter tais modelos. Considerando esta situaçãorelatada, o uso de um modelo confeccionado com esponja, de espuma sintética, com uma camada de material de moldagem denominada de "elastômero". Algo simples, fácil de ser encontrado, de baixo valor aquisitivo, mas que solucionava a questão monetária que poderia inviabilizar a utilização do recurso. (Kumaresan et al., 2013; Uppal et al., 2012; Schijven et al., 2005; Wanibuchi et al., 2016).

A confecção do modelo exibido se destaca pela fácil acessibilidade de espuma e material de elastômero, que em conjunto com a preparação rápida, torna o modelo de sutura adequado quando se procura uma solução de baixo custo. (Kumaresan et al., 2013; Uppal et al., 2012; Schijven et al., 2005).

Desta maneira, podemos observar que os resultados obtidos através do treinamento por simuladores são variados, positivos e demonstram que a aquisição das habilidades a partir destes treinamentos, além de viáveis, é necessária no processo de aprendizado individual de cada profissional. Vale lembrar que constantemente as pesquisas frisam que a simulação é apenas um complemento e não um substituto para o treinamento cirúrgico clínico obrigatório. (Ilburn et al., 2012; Stefanidis et al., 2010; Cohen et al., 2010). 


\section{Considerações Finais}

Os estudos abordados durante esta pesquisa comprovam a eficiência satisfatória dos modelos de simulação descritos pelos pesquisadores consultados. Os modelos facilitam o treinamento de técnicas cirúrgicas pelos profissionais que irão utilizar-se dos procedimentos citados no cotidiano de seu ofício.

Posteriormente, os recursos exemplificados neste estudo devem reduzir o tempo de cada ação executada, o que refletirá diretamente em uma melhor execução das habilidades aprendidas na realidade, propiciando segurança tanto ao profissional, que terá familiaridade no momento do uso de cada técnica, quanto ao paciente que perceberá total domínio situacional sobre os procedimentos que lhe estão sendo aplicados.

\section{Referências}

Ahmed, N., et al (2018). Simulation-based training in maxillofacial surgery: are we going to be left behind? Br J Oral Maxillofac Surg., 57(1):67-71.

Carvalho, F. S. R., et al (2018). Development of Education Material for oral and Maxillofacial Training. J. Maxillofac. Oral Surg, 18(1):155-156.

Cohen, E. R., et al (2010). Cost savings from reduced catheter-related bloodstream infection after simulation-based education for residents in a medical intensive care unit.. Simulation in Healthcare, 5 (2), 98-102.

Ilburn, J. A., et al (2012). Introduction, availability and role of simulation in surgical education and training: Review of current evidence and recommenations from the Association of Surgeons in Training.Int J Surgery., 10(8):393-8.

Kolozsvari, N. O., et al (2011). Sim one, do one, teach one: considerations in designing training curricula for surgical simulation. Journal of Surgical Education, 68(5):421-7.

Kumaresan, R., et al (2013). An Inexpensive Suturing Training Model. J. Oral Maxillofac. Surg., 13(4):609-11.

Lambrecht, J. T., et al (1990).Individual skull model fabrication for craniofacial surgery. Cleft Palate J., 27(4):382-5.

Lambrecht, J. T. H., et al (2010). Haptic model fabrication for undergraduate and postgraduate teaching. Int. J. Oral Maxillofac. Surg., 39(12): 1226-1229.

McCaslin, A. F., et al (2011). Enhancing the Utility of Surgical Simulation: From Proficiency to Automaticity. World Neurosurgery, $76(6)$ :482-4.

O'Callaghan, J., et al (2017). Cross-sectional study of the financial cost of training to the surgical trainee in the UK and Ireland. BMJ Open, 7: e018086.

Pereira, A. S., et al. (2018). Metodologia da pesquisa científica. UFSM. https://repositorio.ufsm.br/bitstream/handle/1/15824/Lic_Computacao_MetodologiaPesquisa-Cientifica.pdf?sequence $=1$

Ploch, C. C., et al (2016). Using 3D Printing to Create Personalized Brain Models for Neurosurgical Training and Preoperative Planning. Neurosurg word, 90:668-674.

Schijven, M. P., et al (2005).SengThe Eindhoven laparoscopic cholecystectomy training course - improving operating room performance using virtual reality training: results from the first E.A.E.S. accredited virtual reality trainings curriculum. Surg Endosc., 19(9):1220-6.

Stefanidis, D., et al (2010).Initial laparoscopic basic skills training shortens the learning curve of laparoscopic suturing and is cost-effective. $J$ Am Coll Surg., 210(4):436-40.

Uppal, N., et al (2011). Low-cost suturing trainging model for use in developing nations. Br J Oral Maxillofac Surg., 50(1):e13-4.

Wanibuchi, H., et al (2016). Training for Skull Base Surgery with a Colored Temporal Bone Model Created by Three-Dimensional Printing Technology. World Neurosurg, 91:66-72.

Wen, G., et al (2016). A practical 3D printed simulator for endoscopic endonasal transsphenoidal surgery to improve basic operational skills. Childs Nerv Syst., 32(6):1109-16.

Werz, S. M., et al (2018). 3D Printed Surgical Simulation Models as education tool by maxillofacial surgeons. Eur J Dent Educ., $22(3)$ )e500-e505. 